\title{
Toxoplasmosis-Associated Secondary Hemophagocytic Syndrome: A Case Report and Literature Review
}

\author{
Xiang Sun*, Jiakui Zhang*, Qianling Ye, Fan Wu, Yinwei Li, Qianshan Tao, Qing Zhang, \\ Weiwei Zhu, Huiping Wang, Jinli Zhu, Zhimin Zhai" \\ Department of Hematology, Hamatological Research Center, The Second Affiliated Hospital of Anhui Medical University, Hefei, \\ China \\ Email: "zzzm889@163.com
}

How to cite this paper: Sun, X., Zhang, J.K., Ye, Q.L., Wu, F., Li, Y.W., Tao, Q.S., Zhang, Q., Zhu, W.W., Wang, H.P., Zhu, J.L. and Zhai, Z.M. (2020) Toxoplasmosis-Associated Secondary Hemophagocytic Syndrome: A Case Report and Literature Review. Open Journal of Blood Diseases, 10, 61-70.

https://doi.org/10.4236/ojbd.2020.103008

Received: June 19, 2020

Accepted: August 1, 2020

Published: August 4, 2020

Copyright $\odot 2020$ by author(s) and Scientific Research Publishing Inc. This work is licensed under the Creative Commons Attribution International License (CC BY 4.0).

http://creativecommons.org/licenses/by/4.0/ (c) (i) Open Access

\begin{abstract}
Background: Toxoplasmosis is an extremely rare disease that occurs in the hosts contact with cat and dog frequently. Most human acute infections go unnoticed in immunocompetent individuals, and gradually transformed into chronic infection. However, while host immunity significantly waned, the risk of reactivation of chronic toxoplasma infection is greatly increased. Reactivation of latent toxoplasmic infection often presents with fever, leukopenia, thrombocytopenia, neurological signs and pneumonia. However, for the non-specific clinical and biological signs and its fetal outcome, toxoplasmosis is often misdiagnosed and only revealed at autopsy. Case Presenation: We report a case hospitalized for a week history of cough, anorexia and fatigue with nasal bleeding for a day. After hospitalization, broad-spectrum antibiotherapy was started without a clear diagnosis of infection. Then the patient did a lot of investigations to search the cause of infection, but there were no positive findings. However, an unexpected discovery was detected from peripheral blood smears, shows $1-3 \mu \mathrm{m}$ in diameter, scattered, short and bow like microorganisms on Day 39 of hospitalization. Combined with the medical history and clinical manifestation, toxoplasmosis was diagnosed finally. Unfortunately, secondary hemophagocytic syndrome (HPS) was diagnosed only two days after targeted anti-infection therapy, and the patient died on Day 45 of hospitalization. Conclusions: Patient with unexplained long-term fever and neurological symptoms, interstitial pneumonitis or myocarditis, toxoplasmosis should be positively considered. Only early diagnosis and treatment can increase the possibility of a successful cure and avoid other secondary diseases.
\end{abstract}

*These authors contributed equally to this work. 


\section{Keywords}

Toxoplasmosis, Infection, Reactivation, Hemophagocytic Syndrome

\section{Background}

Toxoplasma is an obligate intracellular protozoan that belongs to the phylum Apicomplexa, subclass coccidia [1]. Toxoplasmosis is an extremely rare but aggressive infection disease. Primary infection is usually subclinical, but reactivation of latent disease may lead to fatal infection in immunocompromised patients. The clinical manifestation often presents with fever, leukopenia, thrombocytopenia, neurological signs and pneumonia, but these symptoms are often non-specific [2]. The diagnosis remains difficult and the mortality rate is very high in the absence of specific treatment [3]. In many reported cases, the diagnosis was confirmed by autopsy results finally. Therefore, it is particularly important to get early diagnosis.

Hemophagocytic syndrome (HPS) is an immune disorder characterized by uncontrolled activation of lymphocytes, macrophages, and monocytes, and overgrowth of inflammatory cytokines [4]. Symptoms include persistent fever, pancytopenia, hepatosplenomegaly, and hemophagocytosis in bone marrow, spleen, liver, and lymph nodes. Secondary HPS is mostly caused by infection, malignant tumors, and autoimmune diseases, and the most common infection-related HPS is caused by Epstein-Barr virus (EBV), but uncommon infections, such as toxoplasmosis, are less reported.

Here we reported a patient diagnosed with toxoplasmosis-associated secondary HPS to improve the recognization of the disease.

\section{Case Description}

A 17-year-old girl was admitted due to a week history of cough, anorexia and fatigue with nasal bleeding for a day. These symptoms appeared under no obvious inducement and worsen progressively. She was sent to our hospital by classmates for awareness of trance, slow response and high fever.

On presentation, the patient appeared face of acute ill, blood pressure was $100 / 70 \mathrm{mmHg}$, with $40^{\circ} \mathrm{C}$ fever, headache and impairment of consciousness, denied any other complaints. Physical examination was performed after admission with less cooperative. Positive signs included ecchymosis in both legs, red throat, heart rate 140 times per minute and the spleen was palpated $4 \mathrm{~cm}$ below the ribcage. In laboratory findings (see Table 1), cytopenia was noted with leukopenia of $0.21 \times 10^{9} / \mathrm{L}$, thrombocytopenia of $2 \times 10^{9} / \mathrm{L}$ and anemia of $75 \mathrm{~g} / \mathrm{L}$. C reactive protein (C-RP) of $61.9 \mathrm{mg} / \mathrm{L}$. Lactate dehydrogenase (LDH) of $820 \mathrm{U} / \mathrm{L}$. Liver function, renal function, serum electrolyte, coagulation function, thyroid function, immunoglobulin, complement C3 and C4, triglyceride (TG), urinary human chorionic gonadotrop (HCG) and erythrocyte sedimentation rate (ESR) 
Table 1. Patient characteristics during hospitalization.

\begin{tabular}{ccccccc}
\hline Patient characteristics & Day 1 & Day 10 & Day 20 & Day 30 & Day 42 & Reference value \\
\hline White blood cell $\left(\times 10^{9} / \mathrm{L}\right)$ & 0.21 & 2.01 & 3.47 & 0.61 & 0.27 & $4-10$ \\
Neutrophils $\left(\times 10^{9} / \mathrm{L}\right)$ & 0.03 & 1.41 & 1.91 & 0.39 & 0.09 & $2-7$ \\
Hemoglobin (g/L) & 66 & 73 & 75 & 81 & 46 & $110-150$ (Female) \\
Platelet (×109/L) & 6 & 34 & 205 & 68 & 1 & $100-300$ \\
Fibrinogen (g/L) & 2.23 & 1.76 & & & 0.37 & $1.7-4.0$ \\
PT-INR & 1.12 & 1.13 & & & 1.17 & $0.75-1.30$ \\
D-Dimer (mg/L) & 10.36 & 8.61 & & & 5.65 & $0-0.55$ \\
FDP (ug/mL) & 30.60 & 8.03 & & & 13.00 & $0-5.0$ \\
LDH (U/L) & 820 & & & & & $\leq 280$ \\
TG (mmol/L) & 1.22 & & & & & $0.56-1.74$ \\
C-RP (mg/L) & 61.9 & $<5.0$ & 9.9 & 27.9 & 73.4 & $\leq 5$ \\
Alanine aminotransferase (U/L) & 18 & 24 & 68 & 20 & 47 & $10-40$ \\
Aspartate aminotransferase (U/L) & 65 & 36 & 33 & 27 & 261 & $10-40$ \\
Total bilirubin (umol/L) & 18.5 & 23.9 & 13.6 & 12.0 & 15.3 & $3.4-17.1$ \\
ESR (mm/h) & 15 & 12 & 36 & 2 & 5 & $\leq 10$ \\
Creatine kinase (U/L) & 1209 & 18 & & & & $26-140$ \\
Procalcitonin (ng/ml) & & & 0.121 & 0.105 & & $0-0.046$ \\
\hline
\end{tabular}

Dayl was considered as the day admitted to our hospital.

were all normal. Broad-spectrum antibiotherapy was started immediately, using meropenem and amikacin.

However, fever persisted under anti-infection therapy. We subsequently adjusted the anti-infection program, including minocycline, fosfomycin, cefoperazone shubatan, biapenem, ceftriaxone, levofloxacin and cotrimoxazole. Moreover, we also used anti-viral treatment and blood cell growth factors for nearly four weeks. During the treatment, the patient's blood cells gradually returned to normal, other indicators also gradually improved. However, the fever still remains for more than $39^{\circ} \mathrm{C}$ without obvious chills, cough and expectoration. Though the patient's body temperature can be decreased to normal after glucocorticoid treatment followed with no obvious discomfort. Considering prolonged use of broad-spectrum antibiotics may lead to double infection, prophylactic antifungal therapy was added with fluconazole.

In order to search the cause of infection, an extensive investigation was carried out as follows. Index of rheumatic system such as ANA and ANCA were normal. Etiological examination was all negative, including serology for hepatitis B and C virus, HIV, HTLV-1, brucella, cryptococcus, Q-fever and typhoid bacillus. Serology for EBV was negative for IgG and IgM. Acid-fast bacillus and plasmodium were undetectable. Detection of Shigella and Salmonella in stool culture was negative. Despite doing many times, bacteria could not be cultured 
from sputum, blood and bone marrow at all. Routine, biochemical and Cryptococcus detection of cerebrospinal fluid were all negative. Tri-phasic CT demonstrated little inflammation of lungs, splenomegaly of $16 \mathrm{~cm}$, the liver was enlarged but homogenous with no focal lesions or bile duct dilatation, and no pathological lymphadenopathy was noted.

No abnormal cell group was found in bone marrow specimens of the patient by flow cytometry. In peripheral blood, Treg cells accounts for $0.33 \%$ (reference value $5.23 \% \pm 0.94 \%$ in our lab) of $\mathrm{T} \mathrm{CD} 4^{+}$cells, and $\mathrm{CD} 4 / \mathrm{CD} 8$ ratio was 7.38 (reference value $1.41 \pm 0.31$ in our lab) (see Figure 1). Bone marrow cytologic examination indicated elevation NAP integral, considering the presence of infection. Bone marrow biopsy was mildly hypercellular with expression of all three hematopoietic lineages. The marrow cells had normal chromosomes. 18F-Fluorodeoxyglucose (FDG)-positron emission tomography (PET) revealed multiple intense and heterogeneous FDG uptakes within the bone marrow, and hepatosplenomegaly (see Figure 2).

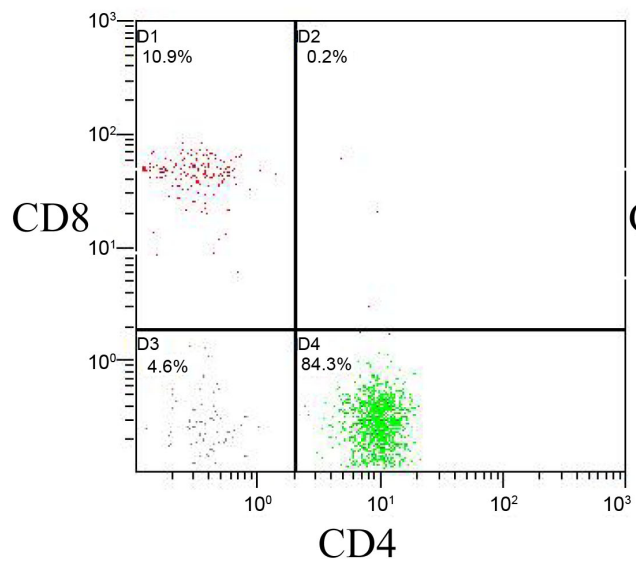

(A)

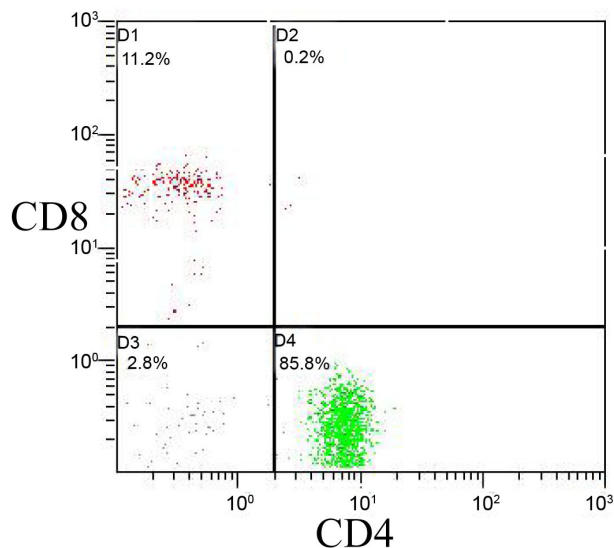

(C)

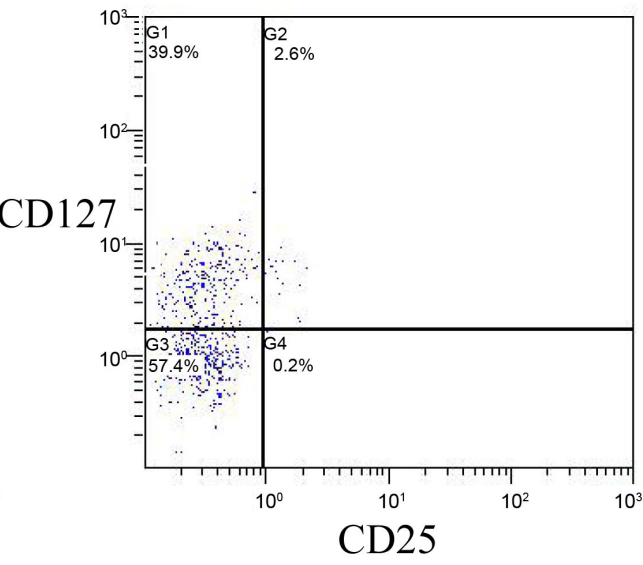

(B)

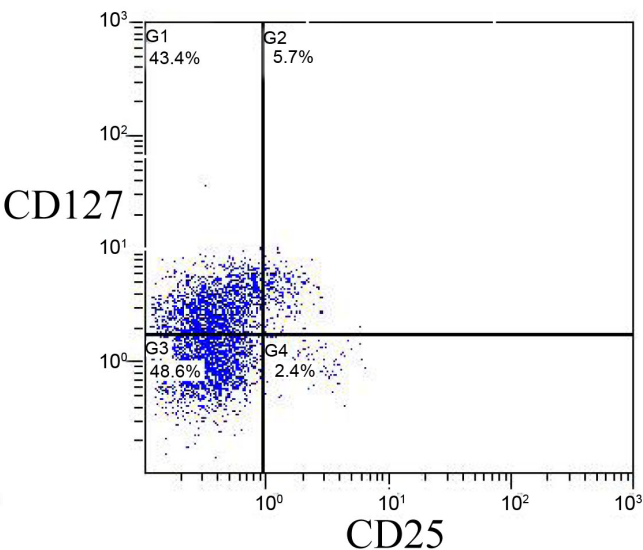

(D)

Figure 1. FCM analysis of peripheral blood specimens from the patient. (A, B) were analyzed on admission, show the CD4/CD8 ratio was 7.73 and the percentage of $\mathrm{CD} 4{ }^{+} \mathrm{CD} 25^{+} \mathrm{CD} 127$ low Treg was $0.2 \%$ within $\mathrm{CD}^{+} \mathrm{T}$ cell gate. $(\mathrm{C}, \mathrm{D})$ were analyzed on Day 24 , show the CD4/CD8 ratio was 7.66 and percentage of $\mathrm{CD} 4{ }^{+} \mathrm{CD} 25^{+} \mathrm{CD} 127$ low Treg was $2.4 \%$ accounted within $\mathrm{CD} 4^{+} \mathrm{T}$ cell gate. 

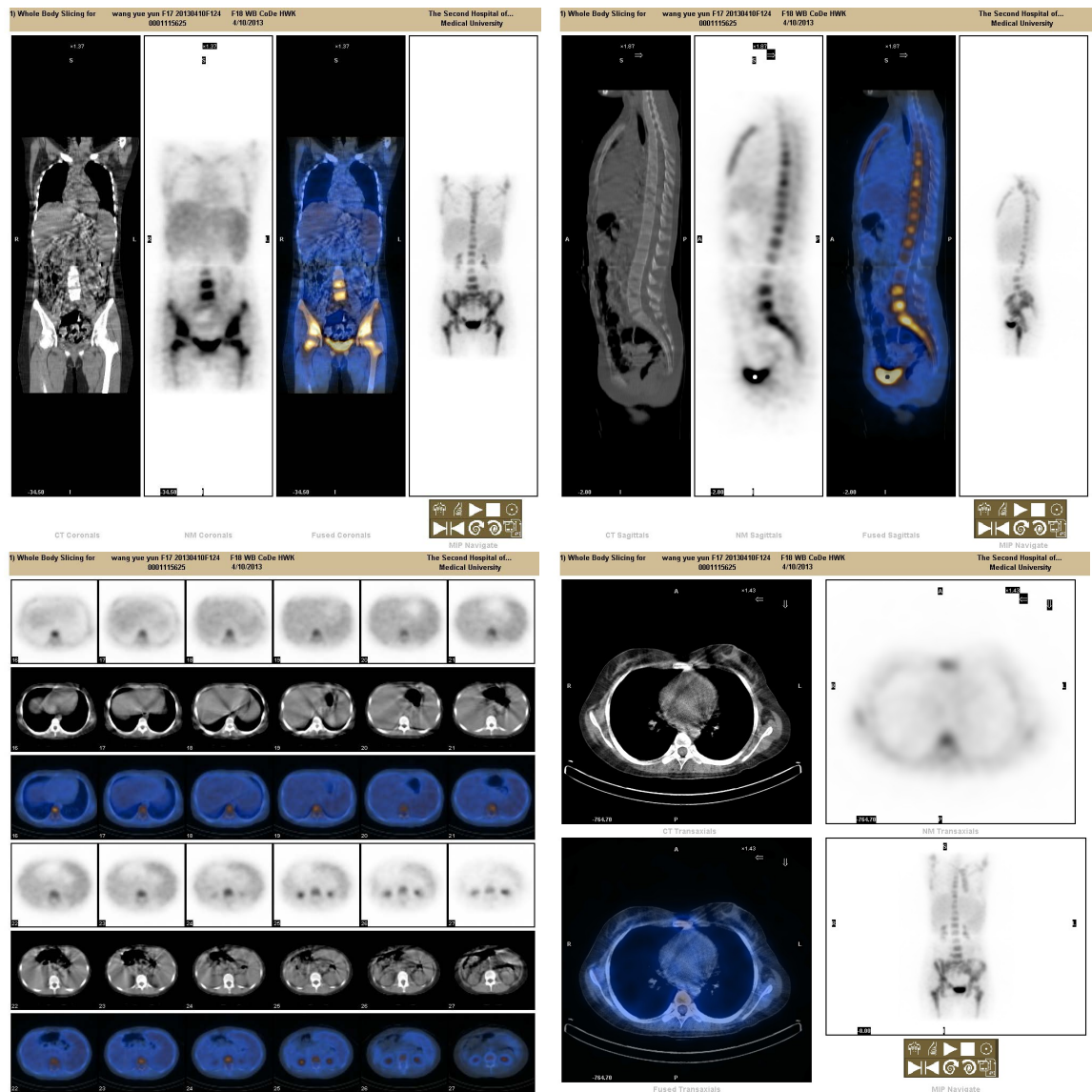

Figure 2. Axial fused FDG-PET image and coronal maximum intensity projection (MIP) image revealing multiple intense and heterogeneous FDG uptakes within the bone marrow in multiple bones of the patient, it also shows the presence of hepatosplenomegaly.

From Day 30, the patient's condition deteriorated with unchanged pancytopenia and worsening liver function tests, and the maximum body temperature raise to about $39.5^{\circ} \mathrm{C}$ again. A third bone marrow aspirate on Day 37 revealed severe hemophagocytosis (see Figure 3). Combined with HLH-2004 diagnostic criteria, the patient fulfilled five of eight diagnostic criteria for hemophagocytic lymphohistiocytosis (HLH) per HLH-2004 [5] (fever, splenomegaly, cytopenias, hypofibrinogenemia and marrow hemophagocytes), so the patient was clearly diagnosed with HPS. In order to find the causative factors of HPS, we again sent out many pathogenic tests.

On Day 39, an unexpected discovery was detected from peripheral blood smears, shows $1-3 \mu \mathrm{m}$ in diameter, scattered, short and bow like microorganisms (see Figure 3). Then we ask the medical history again in detail, found that she had the history of contacting cat and dog before hospitalization. After consulting the relevant literature, we submitted Toxoplasma antibody test and the result indicated that anti-Toxoplasma antibody-IgG was positive. Finally, the patient was diagnosed with toxoplasmosis-associated secondary hemophagocytic syndrome on Day 40.

After a definite diagnosis, targeted anti-infection treatment with azithromycin 


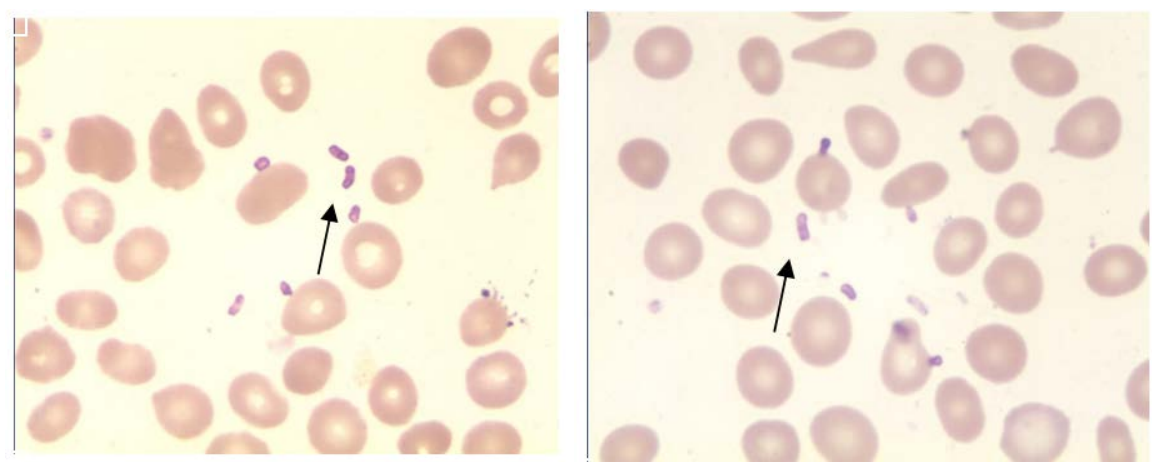

(A)

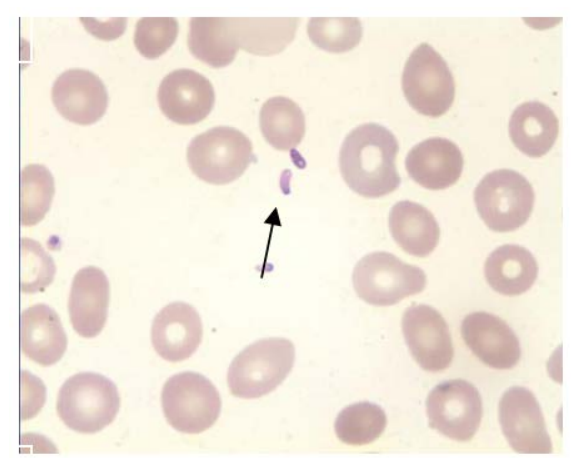

(C)

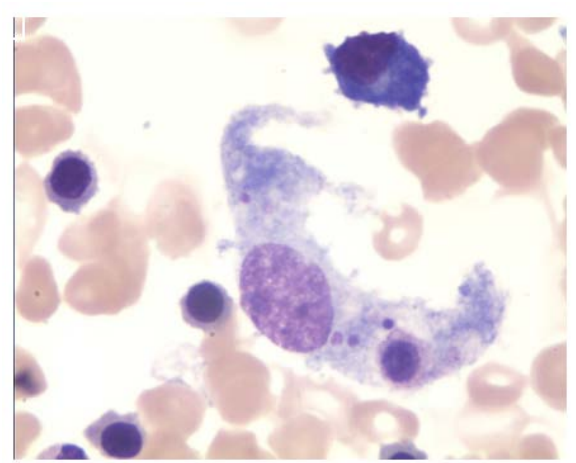

(E)
(B)

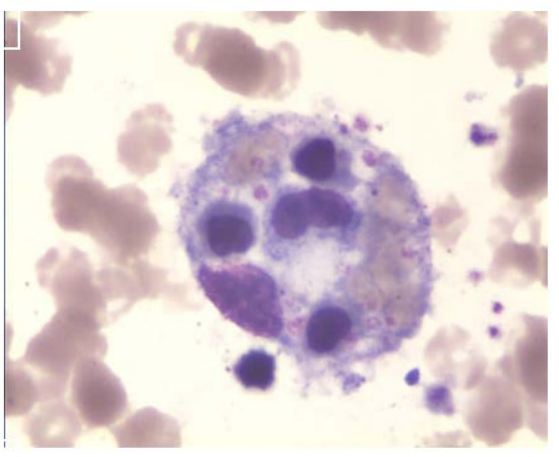

(D)

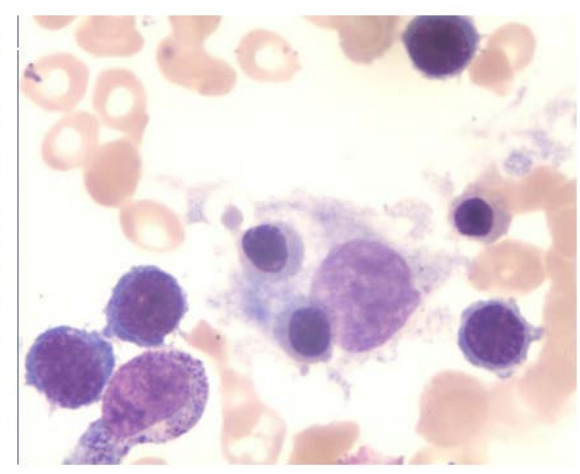

(F)

Figure 3. (A, B, C) Peripheral blood smears results of the patient. This figure shows $1-3$ $\mu \mathrm{m}$ in diameter, scattered, short and bow like microorganisms (Giemsa stain, high power view). (D, E, F) Haemophagocytosis are easy to see in bone marrow smears (Giemsa stain, high power view).

and sulfamethoxazole was started immediately, and meropenem was also used to treat secondary bacterial infection. Supportive treatment such as infusion of blood products and fibrinogen was used as well. However, the efficacy was very poor. Laboratory tests showed worsening liver function, worsening pancytopenia, severe coagulopathy. The patient eventually died of disseminated intravascular coagulation (DIC) on Day 45.

\section{Discussion}

This case indicates the difficulties in diagnosis of toxoplasmosis, and long-term 
fever is the most vexing problems in clinical practice. Meanwhile, it can also be suggested that secondary HPS is often fatal if the original disease was not well controlled.

Human beings can be infected with toxoplasma by ingestion orhandling of raw or undercooked meat (mainly pork and lamb) containing tissue cysts or food or water containing oocysts excreted in the faeces of infected cats. The following acute infection is characterized by rapidly replicating tachyzoite stage parasites that spread widely into host tissues until the infection is controlled by host immune response [6]. Furthermore, most human acute infections go unnoticed in immunocompetent individuals, and gradually transformed into chronic infection with Toxoplasma antibody-IgG positive [7]. However, while host immunity significantly waned, the risk to reactivation of chronic toxoplasma infection is greatly increased [8]. This case report describes a previously healthy girl hospitalized and was diagnosed with acute toxoplasma infection. About two weeks before the admission, the girl fell in love quickly and broke up with great sadness three days before the admission. Meanwhile, she spent every day with her own dog, included eating and sleeping. Therefore, the patient has an incentive to immunosuppression and approach to infect toxoplasma.

In clinical manifestation, acute toxoplasma infection in immunocompromised patients often presented with fever, leukopenia, thrombocytopenia, abnormal liver function. Other symptoms may also occur, such as neurological symptoms, interstitial pneumonitis and myocarditis [9]. Our case was hospitalized with fever, severe thrombocytopenia, elevate AST level and neurological symptoms, including headache and impairment of consciousness. However, all these findings are all nonspecific, this also delayed the correct diagnosis and treatment of the patient. We should carefully ask the patient's medical history, looking for the possibility of specific pathogen infection in patients while long-term antibiotherapy was ineffective.

The method to diagnose toxoplasma infection may be multiple. Toxoplasma can be detected from infected tissue (e.g. liver or lymph nodes biopsies) or from body fluids (e.g. blood, bone marrow aspirate, cerebrospinal fluid). Alternative approach is testing circulating Toxoplasma antigens, by IgM immunoblot assay or by PCR DNA amplification, or by detecting changing titers of specific IgG, IgM, and IgA antitoxoplasma antibodies indirectly [10]. However, for the non-specific clinical and biological signs and its fetal outcome, toxoplasmosis is often misdiagnosed and only revealed at autopsy. In the present case, the clinical features of the patient were in accordance with toxoplasma infection. Furthermore, the high risk factors for toxoplasma infection were also supportive, such as immunosuppression caused by emotional factors and contact with pet frequently. In addition, toxoplasma was detected from blood. Something that needs to be explained here is that at least three times of blood smears were made to exclude the possibility caused by contaminants. We also submitted toxoplasma antibody test and the result indicated that anti-toxoplasma-IgG was positive. The results suggested that the patient had been infected with toxoplasma, and the 
admission is due to reactive toxoplasma infection caused by immunocompromised. In general, the diagnosis of the patient is clear.

Unfortunately, although the patient was eventually diagnosed, secondary HPS was diagnosed two days earlier. HPS is a life-threatening disease that characterized as cytokine release syndrome caused by excessive but non-malignant activation of macrophages and/or histiocytes in bone marrow and other reticuloendothelial systems [11]. Secondary HPS often relate to infection, malignance and autoimmune diseases, and can occur at any age. The currently treatment is always refer to the program recommended by "HLH-2004" international organization [12]. However, the actual effect is not ideal, and the mortality rate is still high.

After diagnosed of toxoplasma infection, targeted anti-infection therapy were started immediately. However, the present case also incorporates secondary HPS, indicates poor prognosis. In a review by Renoult et al. [13], the mortality rate of toxoplasmosis complicating renal transplantation was exceeded $64 \%$. P Y Briand et al. [14] reported that a 12-year-old French child developed secondary hemophagocytic syndrome due to toxoplasma infection and was eventually cured, the cure may be related to the patient's lack of obvious immune suppression and timely treatment against toxoplasma infection. M.-P. Guillaume et al. report a case of an AIDS patient presenting a HPS secondary to an extracerebral form of systemic toxoplasmosis that was only revealed by specific PCR in tissue other than the CNS, and the patient eventually died of ARDS and DIC [15].

In conclusion, patient with unexplained long-term fever and neurological symptoms, interstitial pneumonitis or myocarditis, toxoplasmosis should be positively considered. Only early diagnosis and treatment can increase the possibility of a successful cure and avoid other secondary diseases.

\section{Declarations}

This is an Open Access article which permits unrestricted use, distribution, and reproduction in any medium, provided the original work is properly credited. This applies to the data made available in this article, unless otherwise stated.

\section{Consent}

Written informed consent was obtained from the patient for publication of this Case report and any accompanying images. A copy of the written consent is available for review by the Editor of this journal.

\section{Funding}

This work is funded by the National Natural Science Foundation of China (grant no. 81670179) and the Natural Science Research Project of Anhui Medical University (grant no. KJ2019A0274). The authors thank the patient and his family and all the investigators, including the physicians and laboratory technicians in this study. 


\section{Conflicts of Interest}

The authors declared that they have no competing interests.

\section{References}

[1] Montoya, J.G. and Liesenfeld, O. (2004) Toxoplasmosis. The Lancet, 363, 1965-1976. https://doi.org/10.1016/S0140-6736(04)16412-X

[2] Maschke, M., Dietrich, U., Prumbaum, M., et al. (1999) Opportunistic CNS Infection after Bone Marrow Transplantation. Bone Marrow Transplant, 23, 1167-1176. https://doi.org/10.1038/sj.bmt.1701782

[3] Slavin, M.A., Meyers, J.D., Remington, J.S., et al. (1994) Toxoplasma gondii Infection in Marrow Transplant Recipients: A 20 Year Experience. Bone Marrow Transplant, 13, 549-557.

[4] Lai, W.Y., Wang, Y.N., Wang, J.S., et al. (2018) Epstein-Barr Virus-Associated Hemophagocytic Lymphohistiocytosis in Adults and Adolescents-A Life-Threatening Disease: Analysis of 133 Cases from a Single Center. Hematology, 23, 810-816. https://doi.org/10.1080/10245332.2018.1491093

[5] Henter, J.I., Horne, A., Aricó, M., et al. (2007) HLH-2004: Diagnostic and Therapeutic Guidelines for Hemophagocytic Lymphohistiocytosis. Pediatric Blood \& Cancer, 48, 124-131. https://doi.org/10.1002/pbc.21039

[6] Gazzinelli, R.T., Hakim, F.T., Hieny, S., et al. (1991) Synergistic Role of CD4 ${ }^{+}$and $\mathrm{CD}^{+} \mathrm{T}$ Lymphocytes in IFN-Gamma Production and Protective Immunity Induced by an Attenuated Toxoplasma gondii Vaccine. The Journal of Immunology, 146, 286-292.

[7] Fox, B.A., Rommereim, L.M., Guevara, R.B., et al. (2016) The Toxoplasma gondii Rhoptry Kinome Is Essential for Chronic Infection. MBio, 7, pii: e00193-16.

https://doi.org/10.1128/mBio.00193-16

[8] Contini, C. (2008) Clinical and Diagnostic Management of Toxoplasmosis in the Immunocompromised Patient. Parassitologia, 50, 45-50.

[9] Sumi, M., Aosai, F., Norose, K., et al. (2013) Acute Exacerbation of Toxoplasma gondii Infection after Hematopoietic Stem Cell Transplantation: Five Case Reports among 279 Recipients. International Journal of Hematology, 98, 214-222. https://doi.org/10.1007/s12185-013-1379-8

[10] Segall, L., Moal, M.C., Doucet, L., et al. (2006) Toxoplasmosis-Associated Hemophagocytic Syndrome in Renal Transplantation. Transplant International, 19, 78-80. https://doi.org/10.1111/j.1432-2277.2005.00179.x

[11] Rouphael, N.G., Talati, N.J., Vaughan, C., et al. (2007) Infections Associated with Haemophagocytic Syndrome. Lancet Infectious Diseases, 7, 814-822. https://doi.org/10.1016/S1473-3099(07)70290-6

[12] Schram, A.M. and Berliner, N. (2015) How I Treat Hemophagocytic Lymphohistiocytosis in the Adult Patient. Blood, 125, 2908-2914.

https://doi.org/10.1182/blood-2015-01-551622

[13] Renoult, E., Georges, E., Biava, M.F., et al. (1997) Toxoplasmosis in Kidney Transplant Recipients: Report of Six Cases and Review. Clinical Infectious Diseases, 24, 625-634. https://doi.org/10.1093/clind/24.4.625

[14] Briand, P.Y., Gangneux, J.P., Favaretto, G., et al. (2008) Hemophagocytic Syndrome and Toxoplasmic Primo-Infection. Annales de Biologie Clinique, 66, 199-205.

[15] Guillaume, M.-P., Driessens, N., Libert, M., et al. (2006) Hemophagocytic Syn- 
drome Associated with Extracerebral Toxoplasmosis in an HIV-Infected Patient. European Journal of Internal Medicine, 17, 503-504.

https://doi.org/10.1016/j.ejim.2006.04.008 